\title{
Identification and mobilization of a cryptic antibiotic biosynthesis gene locus from a human-pathogenic Nocardia isolate.
}

Marion Herisse ${ }^{\dagger}$, Keishi Ishida ${ }^{\ddagger}$, Jessica L. Porter ${ }^{\dagger}$, Ben Howden’ Christian Hertweck ${ }^{\ddagger}, *$, Timothy P. Stinear ${ }^{\dagger, l}$ and Sacha J. Pidot ${ }^{\dagger},{ }^{\| *}$

$\dagger$ Department of Microbiology and Immunology, Doherty Institute for Infection and Immunity, University of Melbourne, Melbourne, VIC, 3000, Australia

$\$$ Department of Biomolecular Chemistry, Leibniz Institute, for Natural Product Chemistry and Infection Biology (HKI), Beutenbergstrasse 11a, 07745 Jena, Germany.

1 Microbiological Diagnostic Unit, University of Melbourne, Melbourne, VIC, 3000, Australia

* Natural Product Chemistry, Faculty of Biological Sciences, Friedrich Schiller University Jena, 07743 Jena, Germany.

\section{Table of contents}

Supplementary Table 1. Strains and plasmids used in this study Supplementary Table 2. Primers used in this study. Supplementary Table 3. Nocardia genomes used for type II PKS BGC network analysis. Supplementary Figure 1. Phylogenetic position of CLF proteins from Nocardia type II PKS gene clusters

Supplementary Table 4. ${ }^{1} \mathrm{H}$ and ${ }^{13} \mathrm{C}$ NMR data for brasiliquinone B in $\mathrm{CDCl}_{3}$ Supplementary Figure 2. ${ }^{1} \mathrm{H}$ NMR spectrum of brasiliquinone $\mathrm{B}$ in $\mathrm{CDCl}_{3}$ at $300 \mathrm{~K}$. Supplementary Figure $3 .{ }^{13} \mathrm{C}$ NMR spectrum of brasiliquinone $\mathrm{B}$ in $\mathrm{CDCl}_{3}$ at $300 \mathrm{~K}$. Supplementary Figure 4. ${ }^{1} \mathrm{H}-{ }^{1} \mathrm{H}$ COSY spectrum of brasiliquinone $\mathrm{B}$ in $\mathrm{CDCl}_{3}$ at $300 \mathrm{~K}$ Supplementary Figure 5. HSQC spectrum of brasiliquinone $\mathrm{B}$ in $\mathrm{CDCl}_{3}$ at $300 \mathrm{~K}$. Supplementary Figure 6. HMBC spectrum of brasiliquinone $\mathrm{B}$ in $\mathrm{CDCl}_{3}$ at $300 \mathrm{~K}$ Supplementary Figure 7. The selected ${ }^{1} \mathrm{H}^{1}{ }^{1} \mathrm{H}$ COSY (bold line) and HMBC (arrow) correlations of brasiliquinone B isolated in this study

Supplementary Figure 8. High resolution mass spectrum (negative ion mode) of brasiliquinone B.

Supplementary Figure 9. Proposed pathway for TDP-L-ristosamine biosynthesis from the brq BGC

Supplementary Figure 10. Structures of the brasiliquinone family of antibiotics 
Supplementary Table 1 - Strains and plasmids used in this study

\begin{tabular}{|c|c|c|}
\hline \multicolumn{3}{|l|}{ Strains } \\
\hline Name & Features & Source \\
\hline $\begin{array}{l}\text { Escherichia coli } \\
\text { DH10B }\end{array}$ & $\begin{array}{l}\mathrm{F}^{-} \text {endA1 deo } R^{+} \text {recA1 galE15 galK16 nupG rpsL } \\
\Delta(\text { lac)X74 } 980 \text { lacZAM15 araD139 } \Delta \text { (ara,leu)7697 } \\
\text { mcrA } \Delta\left(\text { mrr-hsdRMS-mcrBC) } \operatorname{Str}^{\mathrm{R}} \lambda^{-}\right.\end{array}$ & Invitrogen \\
\hline $\begin{array}{l}\text { E. coli } \text { ET12567 } \\
(\mathrm{pUZ} 8002)\end{array}$ & $\begin{array}{l}\text { Methylation deficient strain (dam-13::Tn9 dcm-6 } \\
\left.h s d M \mathrm{Cm}^{\mathrm{R}}\right) \text {. pUZ8002 contains tra functions and is } \\
\mathrm{Kan}^{\mathrm{R}} \text {. }\end{array}$ & $\begin{array}{l}\text { Prof. Peter } \\
\text { Leadlay, } \\
\text { Cambridge } \\
\text { University }\end{array}$ \\
\hline $\begin{array}{l}\text { Saccharomyces } \\
\text { cerevisiae VL6-48N }\end{array}$ & $\begin{array}{l}\text { MAT } \alpha, \text { his } 3-D 200, \operatorname{trp} 1-\Delta 1, \text { ura3-41, lys } 2, \text { ade2- } \\
\text { 101, met14, psi+cirO }\end{array}$ & $\begin{array}{l}\text { Dr. Vladimir } \\
\text { Larionov, } \\
\text { National Cancer } \\
\text { Institute, National } \\
\text { Institutes of } \\
\text { Health, Bethesda, } \\
\text { USA }{ }^{1}\end{array}$ \\
\hline $\begin{array}{l}\text { Streptomyces } \\
\text { coelicolor } \mathrm{M} 1152\end{array}$ & $\begin{array}{l}\text { S. coelicolor M145 } \Delta \text { act } \Delta \text { red } \Delta c p k \Delta c d a \\
\text { rpoB[C1298T]) }\end{array}$ & $\begin{array}{l}\text { Prof. Mervyn } \\
\text { Bibb, John Innes } \\
\text { Centre, Norwich, } \\
\text { UK }^{2}\end{array}$ \\
\hline $\begin{array}{l}\text { Nocardia } \\
\text { brasiliensis } \\
\text { AUSMDU00024985 }\end{array}$ & Patient isolate & This study \\
\hline $\begin{array}{l}\text { S. coelicolor M1152 } \\
\text { (pTPS790) }\end{array}$ & $\begin{array}{l}\text { S. coelicolor M1 } 152 \text { containing the } \text { brq } \mathrm{BGC} \\
\text { integrated at the } \Phi C 31 \text { integration site }\end{array}$ & This study \\
\hline
\end{tabular}

\begin{tabular}{|l|l|l|}
\hline Plasmids & \multicolumn{2}{|l|}{} \\
\hline Name & Features & Source \\
\hline pCAP03 & $\begin{array}{l}\text { Used for TAR. URA3 under the control of } P_{\mathrm{ADH}} \cdot \\
\text { Kan }\end{array}$ & $\begin{array}{l}\text { Addgene plasmid } \\
\# 69862^{3}\end{array}$ \\
\hline pCAP03-hyg & $\begin{array}{l}\text { Used for TAR. URA3 under the control of } P_{\mathrm{ADH}} \\
\text { Hyg } \\
\text { sites to replace } \text { aac }(3) I V .\end{array}$ & This study \\
\hline pTPS790 & $\begin{array}{l}\text { pCAP03-hyg containing 55 kb cloned region of } N . \\
\text { brasiliensis AUSMDU00024985 genome containing } \\
\text { the } \text { brq BGC (“24985-06"). Constructed using TAR. }\end{array}$ & This study \\
\hline
\end{tabular}


Supplementary Table 2 - Primers used in this study. Capital letters indicate include restriction enzyme sites.

\begin{tabular}{|c|c|c|}
\hline Name & Sequence & Use \\
\hline $\begin{array}{l}\text { Hyg-F- } \\
\text { NruI }\end{array}$ & aattTCTCGAcgcagcagcgggettcgg & $\begin{array}{l}\text { For cloning hyg into } \\
\text { pCAP03. NruI site at } \\
5 \text {, end }\end{array}$ \\
\hline $\begin{array}{l}\text { Hyg-R- } \\
\text { BglII }\end{array}$ & aattAGATCTCccgtagagattggcgatc & $\begin{array}{l}\text { For cloning hyg into } \\
\text { pCAP03. BglII site at } \\
5 \text {, end. }\end{array}$ \\
\hline $\begin{array}{l}24985-06 \\
\text { capture } \\
\text { fragment }\end{array}$ & $\begin{array}{l}\text { catggtataaatagtggcaatcatgacacgaaatgcgcggcatc } \\
\text { ggattcccagtccactggggaggtttaaacaagcgacggttcc } \\
\text { gcgetcggggccttgtactgttctcgtcggcgtagggtatgtcgaaagctacata }\end{array}$ & $\begin{array}{lr}\text { Inserted by } & \text { Gibson } \\
\text { cloning } & \text { into } \\
\text { XhoI/NdeI } & \text { digested } \\
\text { pCAP03-hyg } & \end{array}$ \\
\hline
\end{tabular}

Supplementary Table 3. Nocardia genomes used for type II PKS BGC network analysis.

\begin{tabular}{|l|l|}
\hline Strain name & Accession number \\
\hline Nocardia abscessus NBRC 100374 & BAFP01000089.1 \\
\hline Nocardia acidivorans NBRC 108247 & BDAW01000008.1 \\
\hline Nocardia africana NBRC 100379 & BDAV01000017.1 \\
\hline Nocardia alba NBRC 108234 & BDAX01000002.1 \\
\hline Nocardia amamiensis NBRC 102102 & BDBA01000003.1 \\
\hline Nocardia amikacinitolerans NBRC 108937 & BDAU01000001.1 \\
\hline Nocardia anaemiae NBRC 100462 & BDAZ01000020.1 \\
\hline Nocardia aobensis NBRC 100429 & BAFQ01000268.1 \\
\hline Nocardia araoensis NBRC 100135 & BAFR01000155.1 \\
\hline Nocardia arthritidis AUSMDU00012717 & PRJNA572075 \\
\hline Nocardia arthritidis NBRC 100137 & BDBB01000001.1 \\
\hline Nocardia asiatica NBRC 100129 & BAFS01000210.1 \\
\hline Nocardia asteroides NBRC 15531 & BAFO02000039.1 \\
\hline Nocardia beijingensis NBRC 16342 & BDBC01000010.1 \\
\hline Nocardia brasiliensis ATCC 700358 & CP003876.1 \\
\hline Nocardia brasiliensis AUSMDU00024985 & PRJNA570883 \\
\hline Nocardia brasiliensis IFM 10847 & BAUA01000170.1 \\
\hline Nocardia brasiliensis NBRC 14402 & BAFT02000115.1 \\
\hline Nocardia brevicatena NBRC 12119 & BAFU01000248.1 \\
\hline Nocardia caishijiensis NBRC 108228 & BDBE01000025.1 \\
\hline Nocardia carnea NBRC 14403 & BAFV01000126.1 \\
\hline Nocardia carnea strain NRRL B-1997 & JNZF01000001.1 \\
\hline Nocardia cerradoensis NBRC 101014 & BAFW01000146.1 \\
\hline Nocardia concava NBRC 100430 & BAFX01000098.1 \\
\hline Nocardia coubleae NBRC 108252 & BDBD01000004.1 \\
\hline
\end{tabular}




\begin{tabular}{|c|c|}
\hline Nocardia crassostreae & BDCH01000015.1 \\
\hline Nocardia cummidelens NBRC 100378 & BDBG01000001.1 \\
\hline Nocardia cyriacigeorgica GUH-2 & FO082843.1 \\
\hline Nocardia cyriacigeorgica NBRC 100375 & BAFY01000328.1 \\
\hline Nocardia elegans NBRC 108235 & \begin{tabular}{|l|} 
BDBF01000013.1 \\
\end{tabular} \\
\hline Nocardia exalbida NBRC 100660 & BAFZ01000164.1 \\
\hline Nocardia farcinica IFM 10152 & AP006618.1 \\
\hline Nocardia farcinica NBRC 15532 & BDBJ01000001.1 \\
\hline Nocardia farcinica NCTC11134 & LN868938.1 \\
\hline Nocardia flavorosea NBRC 108225 & BDCG01000001.1 \\
\hline Nocardia fusca NBRC 14340 & \begin{tabular}{|l|} 
BDCU01000001.1 \\
\end{tabular} \\
\hline Nocardia gamkensis NBRC 108242 & BDBM01000010.1 \\
\hline Nocardia grenadensis NBRC 108939 & BDCJ01000001.1 \\
\hline Nocardia harenae NBRC 108248 & BDBI01000024.1 \\
\hline Nocardia higoensis NBRC 100133 & BAGA01000102.1 \\
\hline Nocardia ignorata NBRC 108230 & BDBI01000001.1 \\
\hline Nocardia inohanensis NBRC 100128 & BDBK01000001.1 \\
\hline Nocardia jejuensis NBRC 103114 & BDBU01000001.1 \\
\hline Nocardia jiangxiensis NBRC 101359 & BAGB01000174.1 \\
\hline Nocardia jinanensis NBRC 108249 C3352 & LNDA01000061.1 \\
\hline Nocardia kruczakiae NBRC 101016 & BDBL01000015.1 \\
\hline Nocardia lijiangensis NBRC 108240 & BDBP01000001.1 \\
\hline Nocardia mexicana NBRC 108244 & BDBV01000017.1 \\
\hline Nocardia mikamii NBRC 108933 & BDCM01000026.1 \\
\hline Nocardia miyunensis NBRC 108239 & BDBQ01000001.1 \\
\hline Nocardia niigatensis NBRC 100131 & \begin{tabular}{|l|} 
BAGC01000114.1 \\
\end{tabular} \\
\hline Nocardia niwae NBRC 108934 & \begin{tabular}{|l|} 
BDCK01000001.1 \\
\end{tabular} \\
\hline Nocardia nova NBRC 15556 & BDBN01000020.1 \\
\hline Nocardia nova SH22a & CP006850.1 \\
\hline Nocardia otitidiscaviarum IFM 11049 & BATZ01000064.1 \\
\hline Nocardia otitidiscaviarum NBRC 14405 & BAGD01000081.1 \\
\hline Nocardia paucivorans NBRC 100373 & BAGE01000052.1 \\
\hline Nocardia pneumoniae NBRC 100136 & BAGF01000059.1 \\
\hline Nocardia pseudobrasiliensis & BBXO01000024.1 \\
\hline Nocardia pseudobrasiliensis NBRC 108224 & BDBS01000022.1 \\
\hline Nocardia pseudovaccinii NBRC 100343 & BDBY01000001.1 \\
\hline Nocardia puris NBRC 108233 & BDBW01000001.1 \\
\hline Nocardia rhamnosiphila NBRC 108938 & BDCL01000001.1 \\
\hline Nocardia rhamnosiphila NBRC 108938 & JOEJ01000001.1 \\
\hline Nocardia salmonicida NBRC 13393 & \begin{tabular}{|l|} 
BDBR01000001.1 \\
\end{tabular} \\
\hline Nocardia seriolae N-2927 & BAWD02000312.1 \\
\hline Nocardia seriolae strain ZJ0503 & JNCT01000001.1 \\
\hline Nocardia seriolae U-1 & BBYQ01000001.1 \\
\hline
\end{tabular}




\begin{tabular}{|l|l|}
\hline Nocardia shimofusensis NBRC 100134 & BDBT01000001.1 \\
\hline Nocardia sienata NBRC 100364 & BDBX01000001.1 \\
\hline Nocardia soli NBRC 100376 & BDCB01000001.1 \\
\hline Nocardia sp. 348MFTsu5.1 & KB911509.1 \\
\hline Nocardia sp. 852002-51244_SCH5132740 & LZSP01000177.1 \\
\hline Nocardia sp. BMG111209 & KB907307.1 \\
\hline Nocardia sp. BMG51109 D892DRAFT & JAFQ01000004.1 \\
\hline Nocardia sp. CNY236 K940DRAFT & AXVD01000007.1 \\
\hline Nocardia sp. NRRL S-836 P437 & LGDY01000106.1 \\
\hline Nocardia sp. NRRL WC-3656 & JOJF01000011.1 \\
\hline Nocardia sp. Root136 & LMFE01000008.1 \\
\hline Nocardia sp. W9405 & LGYZ01000001.1 \\
\hline Nocardia speluncae NBRC 108251 & BDBZ01000001.1 \\
\hline Nocardia takedensis NBRC 100417 & BAGG01000259.1 \\
\hline Nocardia tenerifensis NBRC 101015 & BAGH01000602.1 \\
\hline Nocardia terpenica AUSMDU00012715 & PRJNA573535 \\
\hline Nocardia terpenica NBRC 100888 & BAGI01004372.1 \\
\hline Nocardia terpenica strain IFM 0406 & KV411303.1 \\
\hline Nocardia testacea NBRC 100365 & BAGJ01000274.1 \\
\hline Nocardia thailandica NBRC 100428 & BAGK01000312.1 \\
\hline Nocardia transvalensis NBRC 15921 & BAGL01000127.1 \\
\hline Nocardia uniformis NBRC 13702 & BDCE01000001.1 \\
\hline Nocardia vaccinii NBRC 15922 & BDCC01000001.1 \\
\hline Nocardia vermiculata NBRC 100427 & BDCA01000001.1 \\
\hline Nocardia veterana NBRC 100344 & BAGM01000210.1 \\
\hline Nocardia vinacea NBRC 16497 & BAGN01000158.1 \\
\hline Nocardia violaceofusca NBRC 14427 & BDCN01000031.1 \\
\hline Nocardia vulneris NBRC108396 & BDC101000004.1 \\
\hline Nocardia vulneris strain W9851 & JNFP01000001.1 \\
\hline Nocardia xishanensis NBRC 101358 & BDCF01000001.1 \\
\hline Nocardia yamanashiensis NBRC 100130 & BDCD01000004.1 \\
\hline
\end{tabular}






Supplementary Figure 1. Phylogenetic position of CLF proteins from Nocardia type II PKS gene clusters compared with a set of known gene clusters (see Materials and Methods for further detail). This tree is a labelled version of the same tree that appears in Fig 1. Nodes without numbers have $>75 \%$ bootstrap support. 
Supplementary Table S4. ${ }^{1} \mathrm{H}$ and ${ }^{13} \mathrm{C}$ NMR data for brasiliquinone B in $\mathrm{CDCl}_{3}$.

\begin{tabular}{|c|c|c|c|c|}
\hline \multirow[b]{2}{*}{ position } & \multicolumn{2}{|l|}{$\begin{array}{l}\text { Brasiliquinone B } \\
\text { (this study) }\end{array}$} & \multicolumn{2}{|l|}{ Brasiliquinone B } \\
\hline & ${ }^{1} \mathrm{H}(J=\mathrm{Hz})$ & ${ }^{13} \mathrm{C}$ (mult) & ${ }^{1} \mathrm{H}(J=\mathrm{Hz})$ & ${ }^{13} \mathrm{C}$ (mult) \\
\hline 1 & & $198.4(\mathrm{~s})$ & & $197.6(\mathrm{~s})$ \\
\hline \multirow[t]{2}{*}{2} & $2.49(\mathrm{dd} 16.2,11.1)$ & $45.5(\mathrm{t})$ & $2.49(\mathrm{dd} 16.2,10.8)$ & $45.5(\mathrm{t})$ \\
\hline & $2.99(\mathrm{ddd} 16.2,5.8,1.7)$ & & $3.00($ ddd $16.2,5.6,1.5)$ & \\
\hline 3 & $2.19(\mathrm{~m})$ & $36.6(d)$ & $2.19(\mathrm{~m})$ & $36.5(d)$ \\
\hline \multirow[t]{2}{*}{4} & $2.59(\mathrm{dd} 16.0,11.0)$ & $36.5(\mathrm{t})$ & $2.60(\mathrm{dd} 15.8,10.9)$ & $36.7(\mathrm{t})$ \\
\hline & $2.93(\mathrm{~m})$ & & $2.93(\mathrm{dd} 15.8,2.7)$ & \\
\hline $4 a$ & & $153.3(\mathrm{~s})$ & & $153.3(\mathrm{~s})$ \\
\hline 5 & $7.00(\mathrm{~s})$ & $121.1(\mathrm{~d})$ & $7.00(\mathrm{~s})$ & $116.6(d)$ \\
\hline 6 & $12.29(\mathrm{OH}, \mathrm{s})$ & $163.4(\mathrm{~s})$ & $12.38(\mathrm{OH}, \mathrm{s})$ & $162.5(\mathrm{~s})$ \\
\hline $6 a$ & & $116.6(\mathrm{~s})$ & & $115.1(\mathrm{~s})$ \\
\hline 7 & & $192.5(\mathrm{~s})$ & & $192.6(\mathrm{~s})$ \\
\hline $7 \mathrm{a}$ & & $115.0(\mathrm{~s})$ & & $121.2(\mathrm{~s})$ \\
\hline 8 & $11.69(\mathrm{OH}, \mathrm{s})$ & $162.0(\mathrm{~s})$ & $11.69(\mathrm{OH}, \mathrm{s})$ & $163.5(\mathrm{~s})$ \\
\hline 9 & $7.25(\mathrm{~m})$ & $123.9(\mathrm{~d})$ & $7.27(\mathrm{dd} 7.6,1.5)$ & $120.1(\mathrm{~d})$ \\
\hline 10 & $7.66(\mathrm{~m})$ & $137.4(\mathrm{~d})$ & $7.68(\mathrm{t} 7.8)$ & $133.3(\mathrm{~d})$ \\
\hline 11 & $7.66(\mathrm{~m})$ & $120.0(\mathrm{~d})$ & $7.66(\mathrm{dd} 7.8,1.5)$ & $124.0(\mathrm{~d})$ \\
\hline $11 \mathrm{a}$ & & $135.3(\mathrm{~s})$ & & $135.4(\mathrm{~s})$ \\
\hline 12 & & $182.9(\mathrm{~s})$ & & $183.8(\mathrm{~s})$ \\
\hline $12 \mathrm{a}$ & & $137.6(\mathrm{~s})$ & & $135.4(\mathrm{~s})$ \\
\hline $12 b$ & & $130.2(\mathrm{~s})$ & & $128.8(\mathrm{~s})$ \\
\hline 13 & $1.52(\mathrm{~m})$ & $28.7(\mathrm{t})$ & $1.53(\mathrm{~m})$ & $28.8(\mathrm{t})$ \\
\hline 14 & $1.00(\mathrm{t} 7.5)$ & $11.1(\mathrm{q})$ & $1.00(\mathrm{t} 7.4)$ & $11.1(\mathrm{q})$ \\
\hline
\end{tabular}






Supplementary Figure 2. ${ }^{1} \mathrm{H}$ NMR spectrum of brasiliquinone $\mathrm{B}$ in $\mathrm{CDCl}_{3}$ at $300 \mathrm{~K}$.

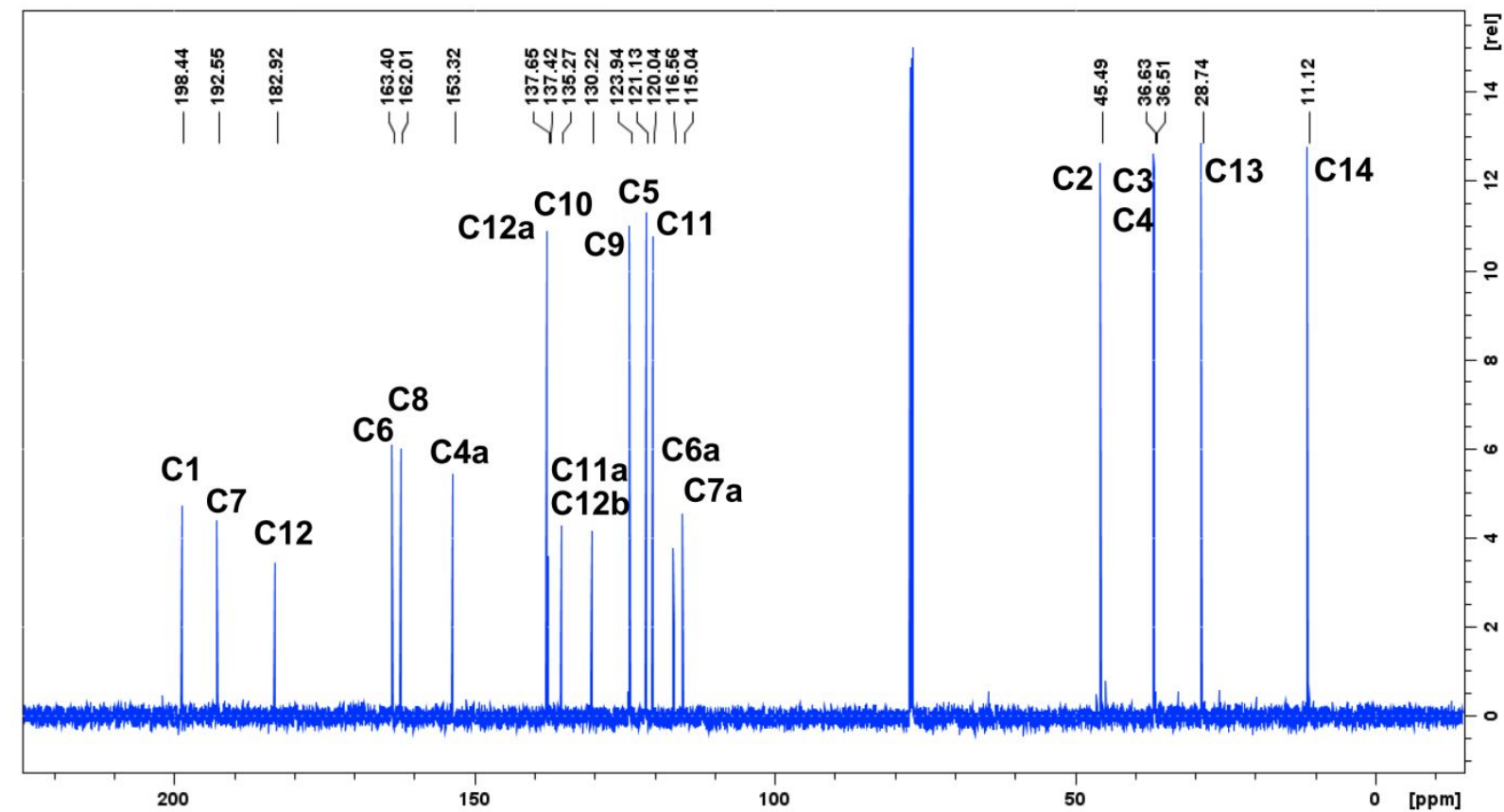

Supplementary Figure 3. ${ }^{13} \mathrm{C}$ NMR spectrum of brasiliquinone $\mathrm{B}$ in $\mathrm{CDCl}_{3}$ at $300 \mathrm{~K}$. 


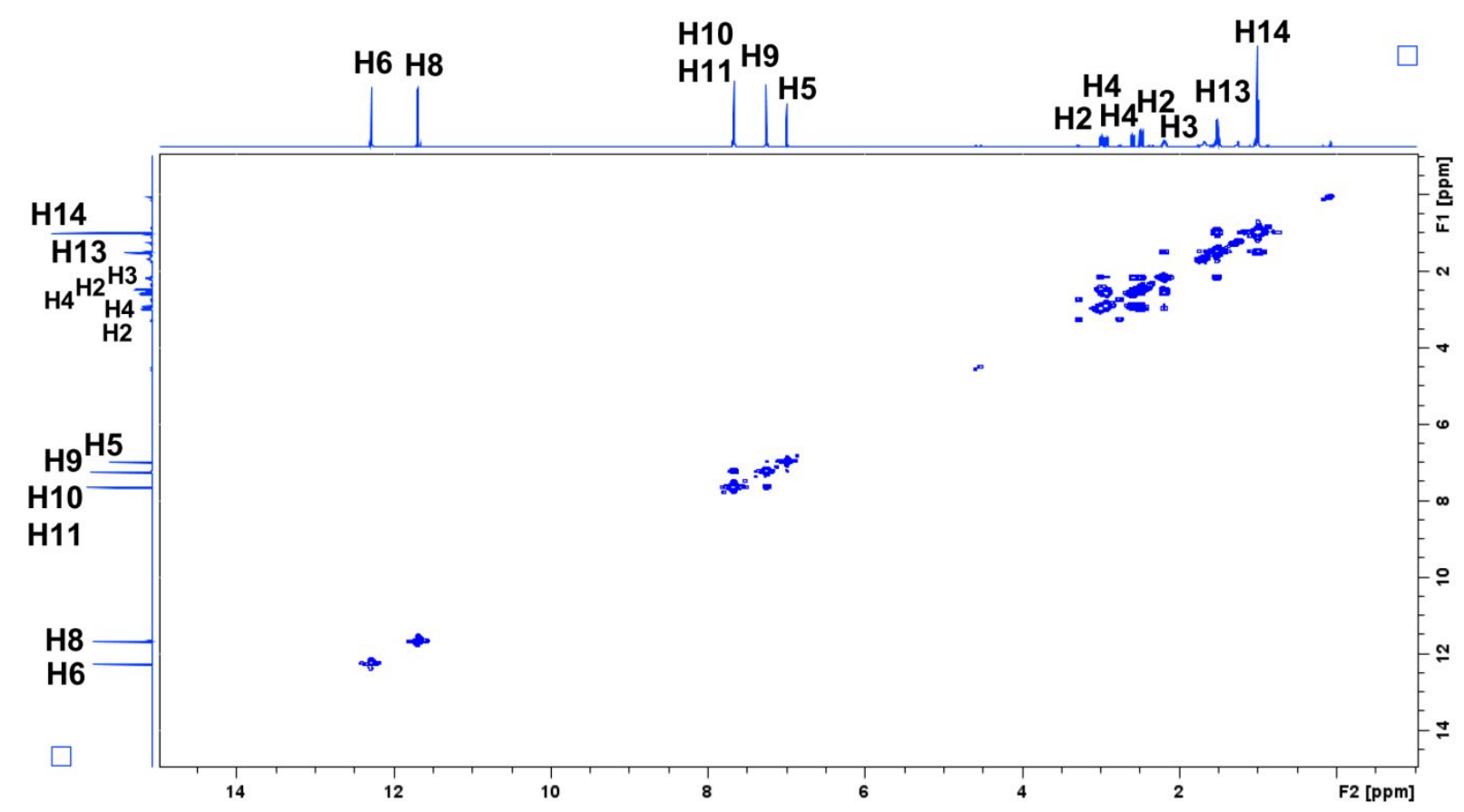

Supplementary Figure 4. ${ }^{1} \mathrm{H}-{ }^{1} \mathrm{H}$ COSY spectrum of brasiliquinone B in $\mathrm{CDCl}_{3}$ at $300 \mathrm{~K}$.

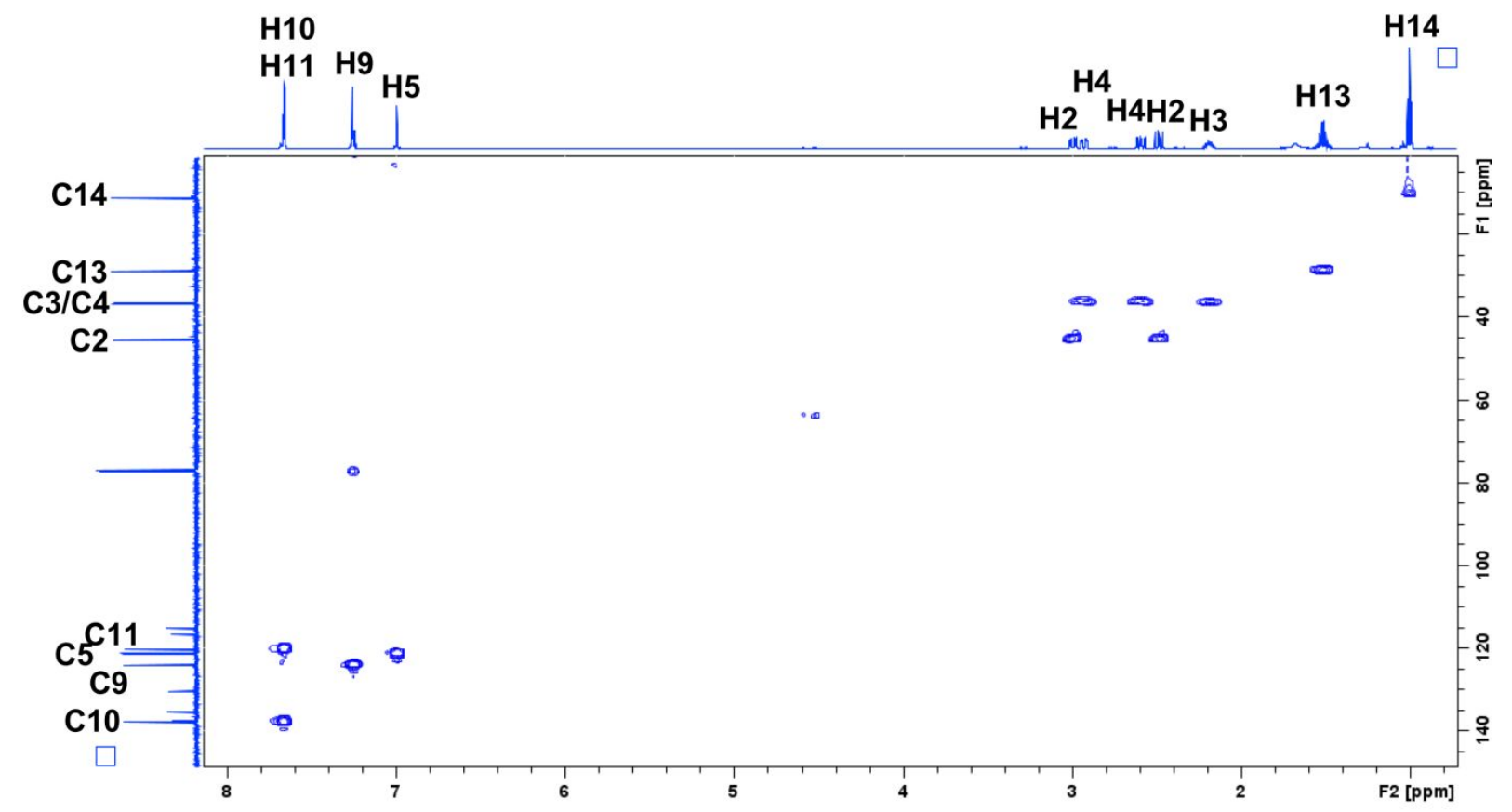

Supplementary Figure 5. HSQC spectrum of brasiliquinone $\mathrm{B}$ in $\mathrm{CDCl}_{3}$ at $300 \mathrm{~K}$. 


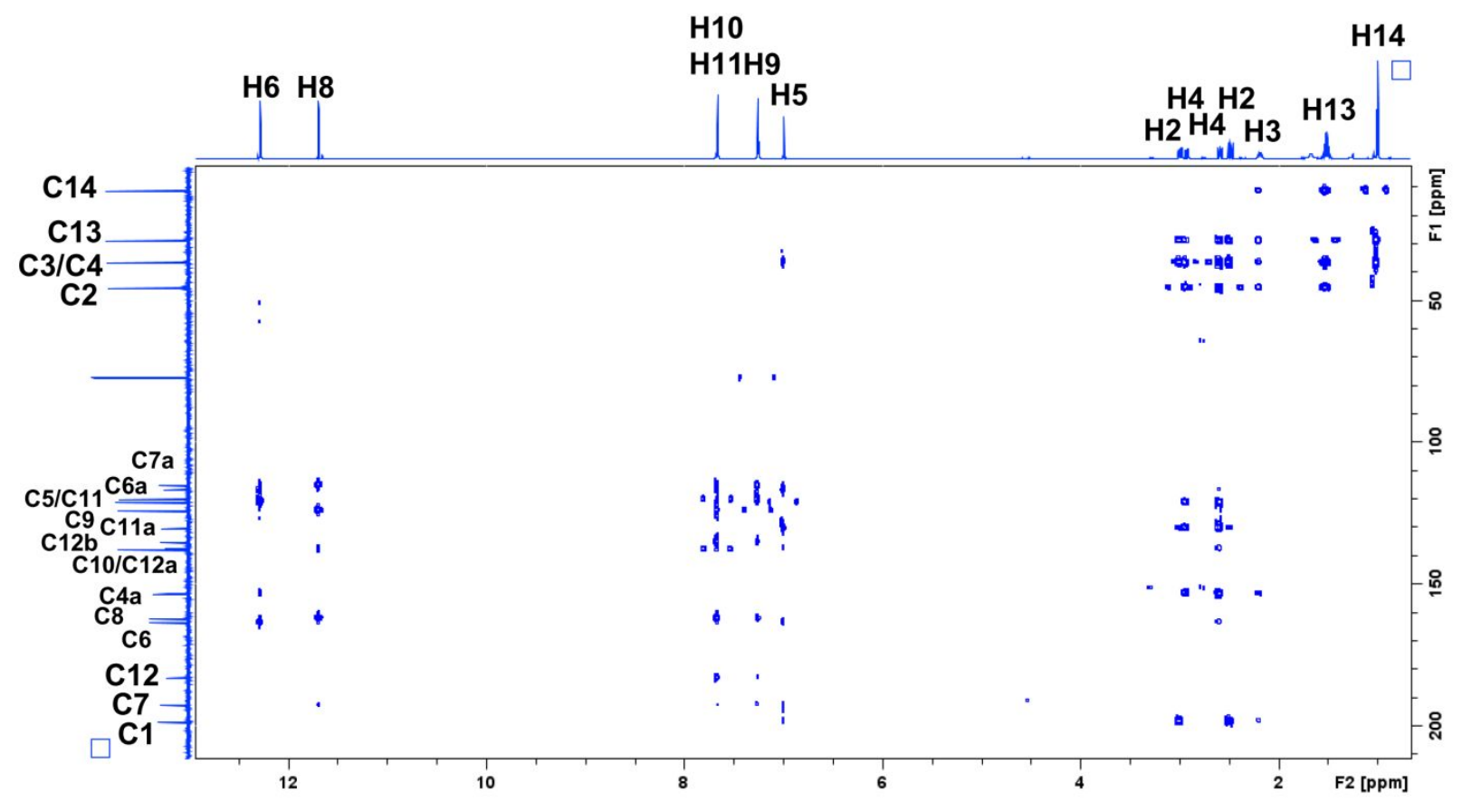

Supplementary Figure 6. $\mathrm{HMBC}$ spectrum of brasiliquinone $\mathrm{B}$ in $\mathrm{CDCl}_{3}$ at $300 \mathrm{~K}$.



Supplementary Figure 7. The selected ${ }^{1} \mathrm{H}-{ }^{1} \mathrm{H}$ COSY (bold line) and HMBC (arrow) correlations of brasiliquinone $\mathrm{B}$ isolated in this study. 


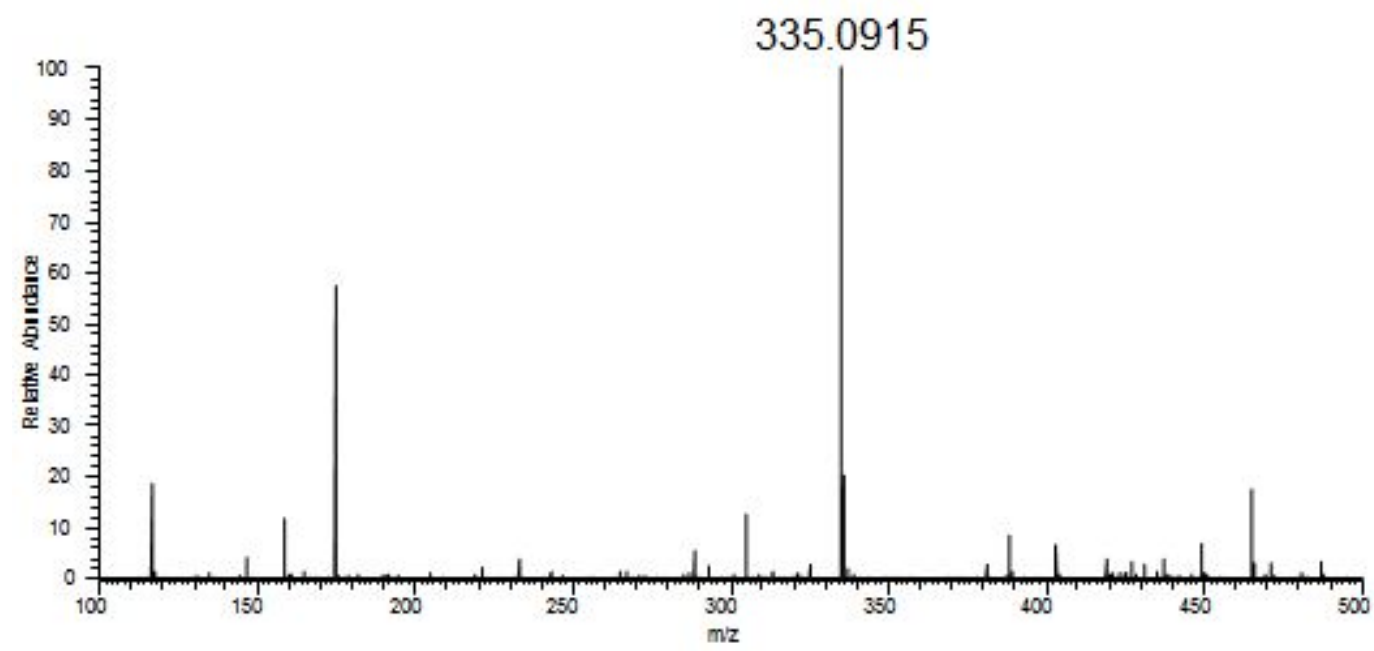

Supplementary Figure 8. High resolution mass spectrum (negative ion mode) of brasiliquinone B.
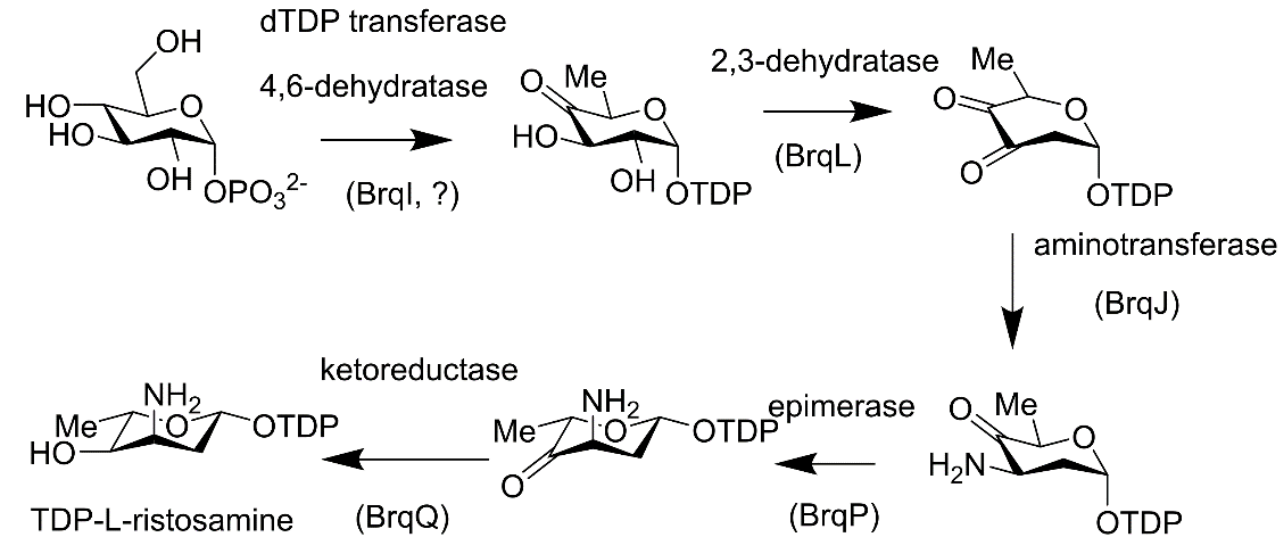

Supplementary Figure 9 - Proposed pathway for TDP-L-ristosamine biosynthesis from the $b r q$ BGC, with predicted proteins involved and their functions labelled at each step. 
<smiles>CCC1CC(=O)c2c(cc(O)c3c2C(=O)c2cccc(OC4C[C@H](N)[C@@H](O)[C@H](C)O4)c2C3=O)C1</smiles><smiles>CCC1CC(=O)c2c(cc(O)c3c2C(=O)c2cccc(O)c2C3=O)C1</smiles><smiles>CCC1CC(=O)c2c(cc(O)c3c2C(=O)c2c(OC)cccc2C3=O)C1</smiles>

brasiliquinone $\mathrm{B}$

brasiliquinone $\mathrm{C}$

Supplementary Figure 10 - Structures of the brasiliquinone family of antibiotics.

\section{References}

(1) Kouprina, N.; Larionov, V. Selective Isolation of Genomic Loci from Complex Genomes by Transformation-Associated Recombination Cloning in the Yeast Saccharomyces Cerevisiae. Nat. Protoc. 2008, 3 (3), 371-377. https://doi.org/10.1038/nprot.2008.5.

(2) Gomez-Escribano, J. P.; Bibb, M. J. Engineering Streptomyces Coelicolor for Heterologous Expression of Secondary Metabolite Gene Clusters. Microb Biotechnol 2011, 4 (2), 207 215. https://doi.org/10.1111/j.1751-7915.2010.00219.x.

(3) Tang, X.; Li, J.; Millan-Aguinaga, N.; Zhang, J. J.; O’Neill, E. C.; Ugalde, J. A.; Jensen, P. R.; Mantovani, S. M.; Moore, B. S. Identification of Thiotetronic Acid Antibiotic Biosynthetic Pathways by Target-Directed Genome Mining. ACS Chem. Biol. 2015, 10, 2841-2849. https://doi.org/10.1021/acschembio.5b00658. 